\title{
A REPRESENTAÇÃO LÉXICO-SEMÂNTICA DE ALGUNS TIPOS DE VERBOS MONOARGUMENTAIS
}

\author{
Sergio de Moura Menuzzi* \\ Pablo Nunes Ribeiro**
}

\begin{abstract}
RESUMO: Neste trabalho, defendemos a distinção proposta por Rappaport-Hovav \& Levin (1998, 2010) entre verbos de maneira e verbos de resultado. Contudo, contrariamente à análise das autoras, sustentamos que os verbos de modo de movimento não agentivos (p. ex., rolar, girar, deslizar, etc.) são verbos de maneira, e não de resultado. A proposta das autoras procura explicar ofato de que tais verbos participam de um processo lexical semelhante à alternância causativa, em contraste com verbos de modo de movimento agentivos (p. ex., correr, nadar, caminhar, etc.). Em nossa análise, isto se dá porque a raiz de maneira dos primeiros modifica um predicado primitivo MOVE, não volicional - por isso, mais facilmente associado à causação externa, no que difere do predicado volicional ACT, envolvido nos verbos de modo de movimento agentivos. Mostramos que a semântica do modificador quase fornece suporte à tese de que ambas as classes de verbos de movimento são verbos de maneira.
\end{abstract}

PALAVRAS-CHAVE: semântica lexical - estrutura de eventos - verbos de modo de movimento

ABSTRACT: In this paper, we argue in favor of the distinction proposed by Rappaport Hovav \& Levin $(1998,2010)$ between manner and result verbs. However, against the analysis of the authors, we maintain that non-agentive manner of motion verbs (e.g., roll, twist, slide, etc.) are manner verbs, and not result verbs. The authors' proposal aims to explain the fact that these verbs participate in a lexical process similar to the causative alternation, in contrast with agentive manner of motion verbs (e.g. run, swim, walk, etc.). In our analysis, it is explained by the fact that the root of non-agentive manner of motion verbs modifies the non-volitional primitive predicate MOVE - since it is a non-volitional predicate, it is more easily associated to external causation, differing in that point from the volitional predicate ACT, involved in agentive manner of motion verbs. We show that the semantics of the modifier almost supports the thesis that both classes of manner of motion verbs are manner verbs.

KEYWORDS: lexical semantics - event structure - manner of motion verbs

\section{INTRODUÇÃO}

De acordo com Rappaport Hovav \& Levin (1998, 2010), os verbos não estativos dividem-se em duas grandes classes - verbos de maneira e verbos de resultado. Grosso modo, verbos de maneira são aqueles que especificam em seu significado um modo de realizar uma ação (p. ex., correr, nadar, varrer, etc.), enquanto verbos de resultado especificam um estado resultante (p. ex., congelar, quebrar, abrir, etc.). Conforme as autoras, esta classificação é relevante gramaticalmente, uma vez que estas classes

\footnotetext{
* Doutor em linguística e professor adjunto da Universidade Federal do Rio Grande do Sul (UFRGS). smenuzzi@gmail.com

** Doutorando do Programa de Pós-Graduação em Letras da Universidade Federal do Rio Grande do Sul (UFRGS) / Mestre pela mesma instituição. pablonribeiro@yahoo.com.br
} 
verbais apresentam padrões distintos de realização sintática dos argumentos, incluindo a participação ou não na alternância causativa.

(1) a. Maria quebrou o copo.

b. O copo quebrou.

c. *Maria quebrou (a tarde inteira).

(2) a. João varreu a sala.

b. *A sala varreu.

c. João varreu (a tarde inteira).

Verbos de mudança de estado típicos, como quebrar, constituem a base dos verbos que participam da alternância causativa (cf. (1a,b)), enquanto verbos de maneira, como varrer, não a permitem. Em (2c), podemos observar que varrer permite a omissão de seu objeto direto; entretanto, a forma incoativa - isto é, a forma em que o paciente/tema é sujeito - não é possível (cf. (2b)).

No entanto, apesar de a distinção entre verbos de maneira e de resultado aparentemente ter um papel importante na definição dos verbos que participam ou não da alternância causativa, algumas classes verbais impõem certas dificuldades a esta proposta. Este é o caso, especialmente, da classe dos verbos classificados por Levin \& Rappaport Hovav (1995) como verbos de "modo de movimento não agentivos" (p. ex., rolar, girar, quicar). Estes verbos participam de um processo semelhante à alternância causativa, em contraste com os verbos de modo de movimento agentivos, como nadar, correr e caminhar (ignoramos aqui o uso idiomático de correr, que não significa "fazer correr", cf. \#O treinador correu o João):

(3) a. A bola rolou/girou/quicou.

b. João rolou/girou/quicou a bola.

(4) a. Paulo nadou/correu/caminhou.

b. *Maria nadou/correu/caminhou Paulo.

Conforme Levin e Rappaport-Hovav, os verbos em (4) denotam eventualidades causadas internamente - porque são verbos volitivos, em que o participante encontra-se intencionalmente envolvido no evento - e, por isso, possuem uma representação léxicosemântica monoargumental (cf. (6) abaixo). Tendo uma representação básica monoargumental, não podem participar da alternância causativa: de acordo com as autoras, esta alternância é um processo pelo qual o argumento causador deixa de ser expresso na sintaxe; assim, participam dela apenas verbos que possuem uma estrutura léxico-semântica bi-argumental - isto é, cuja estrutura apresenta um causador externo ao evento de mudança do paciente/tema. 
Levin e Rappaport-Hovav sugerem outra análise para os verbos em (3): estes denotariam eventualidades causadas externamente. Por isso, teriam uma representação léxico-semântica causativa - isto é, um causador externo ao evento de mudança do paciente/tema -, o que possibilitaria sua participação na alternância. A única representação proposta pelas autoras para verbos de causação externa é a ilustrada em (5) abaixo; assim, em sua análise, os verbos de modo de movimento não agentivos teriam uma representação léxico-semântica similar à dos verbos de resultado, como quebrar, e não à dos verbos de maneira, como nadar:

\section{(5) $[[x$ ACT ] CAUSE $[y$ BECOME $<$ RESULT-STATE $>$ ] $]$ \\ (6) $\left[x \mathrm{ACT}_{<M A N N E R>}\right]$}

Esta análise vai contra a intuição de que os verbos de modo de movimento não agentivos - ainda que apresentem um processo semelhante à alternância causativa também são verbos de maneira; esta intuição é tão clara que o próprio nome da classe a revela: são verbos que denotam o modo como o participante envolvido no evento se movimenta. Além disso, crucialmente, os verbos de modo de movimento, em seu uso intransitivo, também denotam atividades dinâmicas - característica dos verbos de maneira, como vemos pelo contraste entre (1) e (2). Na seção 3, utilizaremos o teste com quase para confirmar esta observação.

Neste trabalho, defendemos que é necessária uma análise alternativa à de Levin e Rappaport-Hovav. Esta análise deve dar conta não somente (i) dos padrões de realização sintática dos argumentos destes verbos - em particular, do fato de apresentarem processos lexicais associados à causação, isto é, similares à alternância causativa -, mas também (ii) da intuição de que pertencem à classe dos verbos de maneira.

Em síntese, nossa proposta é a seguinte: (a) como na análise de Rappaport-Hovav \& Levin (2010), a raiz de maneira dos verbos de modo de movimento agentivos modifica o predicado primitivo ACT, que é volicional; (b) em contraste - e diferentemente do que propõem Rappaport Hovav \& Levin -, a raiz de maneira dos verbos de modo de movimento não agentivos modifica outro predicado primitivo, MOVE, não volicional; (c) por envolverem um processo (neste caso, movimento) não volicional e não causado internamente, os eventos denotados por verbos de modo de movimento não agentivos são mais facilmente concebíveis como sendo causados externamente, o que os torna compatíveis lexicalmente, em português brasileiro, com o primitivo CAUSE.

Com esta proposta, podemos vislumbrar uma explicação para as observações fundamentais (i) e (ii) acima. Especificamente:

Com relação a (ii): as hipóteses (a) e (b) são responsáveis por expressar a idéia de que verbos de modo de movimento não agentivos são, afinal, verbos semelhantes aos de 
modo de movimento agentivos - ambas as classes envolvem verbos de maneira. Já com relação a (i): a hipótese (c) é o que deve explicar o fato de que verbos de modo de movimento não agentivos - diferentemente dos verbos de modo de movimento agentivos - participam de um processo lexical semelhante ao da alternância causativa. ${ }^{1}$

Neste trabalho, nosso objetivo estará centrado em (ii) - isto é, nosso interesse aqui é fornecer suporte para a hipótese de que verbos de modo de movimento não agentivos são verbos de maneira, como os verbos de modo de movimento agentivos. O que segue está organizado da seguinte forma: na primeira seção, discutimos mais detalhadamente a base semântica da distinção entre verbos de maneira e de resultado definida por Rappaport Hovav \& Levin (1998, 2010); na segunda seção, apresentamos nossa proposta para a representação léxico-semântica dos verbos de modo de movimento; por fim, analisamos as propriedades semânticas do modificador quase, mostrando que ambas as classes de verbos se comportam de modo similar com relação a ele - e diferentemente dos verbos de mudança de estado.

\section{AS NOÇÕES DE MANEIRA E DE RESULTADO}

A fim de dar conta dos diferentes padrões de realização sintática dos argumentos observados nos verbos não estativos, Rappaport Hovav \& Levin $(1998,2010)$ propõem uma teoria mais articulada das representações léxico-semânticas destes verbos. Nesta teoria, o componente idiossincrático do verbo, ou seja, sua "raiz", e o componente estrutural, que representa o tipo de evento, possuem papéis diferentes: o componente estrutural é composto por funções semânticas primitivas, e o idiossincrático entra como modificador ou argumento destas funções. Especificamente, as autoras formulam um conjunto de regras de realização canônicas que expressam as formas como a categoria ontológica da raiz (p. ex., maneira, instrumento, estado resultante, etc.) pode se integrar à estrutura de eventos básica do verbo: ${ }^{2}$

(7) maneira $\rightarrow\left[x \mathrm{ACT}_{<M A N N E R>}\right]$

(p. ex., correr, morder, rir, etc.)

(8) instrumento $\rightarrow\left[x \mathrm{ACT}_{<I N S T R U M E N T>}\right]$

(p. ex., escovar, martelar, serrar, etc.)

(9) contêiner $\rightarrow[x$ CAUSE $[y$ BECOME AT $<$ CONTAINER $>$ ]]

(p. ex., encaixotar, empacotar, engarrafar, etc.)

(10) estado causado internamente $\rightarrow[x<$ STATE $>]$

(p. ex., florescer, desabrochar, apodrecer, etc.)

\footnotetext{
${ }^{1}$ A nosso ver, trata-se de um processo de "causativização", diferentemente da alternância causativa, que é um processo de "anticausativização". Entretanto, não trataremos deste ponto no presente trabalho.

${ }^{2}$ Os exemplos foram traduzidos do original pelos autores.
} 
(11) causado externamente; ou seja, estado resultante $\rightarrow$

[ [ $x$ ACT ] CAUSE [ $y$ BECOME <RESULT-STATE $>$ ]]

(p. ex., quebrar, secar, derreter, etc.)

(RAPPAPORT-HOVAV \& LEVIN, 2010, p. 24)

Como podemos observar nas regras acima, as raízes podem tanto ser argumentos na representação dos eventos (cf. (9), (10) e (11)), ou podem ser "modificadores" de predicados primitivos (cf. (7) e (8)), como explicam Rappaport Hovav \& Levin (2010). As autoras sustentam que as raízes de maneira modificam ACT, enquanto as de resultado são argumentos de BECOME, sendo que não pode haver raízes que expressam tanto maneira como resultado. Assim, a teoria assume que as raízes que expressam maneira e resultado possuem distribuição complementar nas representações léxicosemânticas dos verbos.

Definida a hipótese da complementaridade entre raízes de maneira e de resultado, Rappaport Hovav \& Levin (2010) procuram especificar melhor a noção de resultado utilizada em sua teoria. Primeiramente, as autoras descartam a ideia de que a noção de resultado possa ser igualada à de telicidade: apesar de a telicidade envolver normalmente um estado resultante, sabe-se que esta noção é codificada lexicalmente apenas em alguns verbos - em regra, a telicidade de um predicado é definida composicionalmente (cf. Filip \& Rothstein, 2006; Krifka, 1998; entre outros). Rappaport Hovav \& Levin (2010) argumentam que a noção de resultado relevante para sua teoria deve ser entendida não em termos de telecidade, mas de "mudança escalar": raízes de resultado especificariam mudanças escalares, enquanto raízes de maneira, mudanças não escalares.

Segundo as autoras, verbos que denotam eventos de mudança escalar especificam uma escala - isto é, um conjunto de pontos que indicam valores diferentes - para um determinado atributo, com uma relação de ordenamento associada. Uma mudança escalar em uma entidade consiste, portanto, em uma mudança nos valores de um determinado atributo em uma direção particular, determinada pelo ordenamento especificado na semântica do verbo. Verbos de mudança de estado (p. ex., aquecer, abrir, secar) seriam exemplos típicos de verbos que especificam mudanças deste tipo. No caso de um verbo como aquecer, a escala está relacionada à temperatura, e o ordenamento é definido em direção aos valores mais altos deste atributo. Entretanto, nem todos os verbos de mudança escalar denotam escalas com múltiplos pontos; há também verbos que denotam escalas de apenas dois pontos, como, por exemplo, o verbo quebrar.

Os verbos que denotam mudanças não escalares são, por sua vez, aqueles que envolvem uma mudança que não pode ser caracterizada em termos de um conjunto ordenado de valores de um atributo. Conforme as autoras, em geral, estes verbos envolvem mudanças complexas, resultantes da combinação de múltiplas mudanças distintas, o que indica que não há uma escala de mudança específica. Por exemplo, o 
evento denotado por correr envolve simplesmente uma sequência de padrões de movimentos, a qual o distingue de outras atividades, como nadar - sendo que estes padrões não representam uma mudança nos valores de um atributo único.

Considerando-se esta distinção semântica, parece claro que verbos de modo de movimento não agentivos, como rolar, rodar e deslizar, são similares aos demais verbos de modo de movimento: denotam eventos de mudança não escalar, pois não lexicalizam nenhum tipo de ordenamento de valores em uma escala. Por este critério, portanto, os verbos de modo de movimento possuiriam uma estrutura de eventos como a expressa em (7) - e, na análise de Rappaport-Hovav \& Levin (2010), não poderiam apresentar a alternância causativa. Mas isso contraria os fatos.

$\mathrm{Na}$ seção seguinte, apresentaremos nossa proposta para a representação léxicosemântica dos verbos de modo de movimento - agentivos e não agentivos. Nela, estes verbos são verbos de maneira, distinguindo-se dos verbos de mudança de estado.

\section{A REPRESENTAÇÃO LÉXICO-SEMÂNTICA DOS VERBOS DE MODO DE MOVIMENTO}

Como mencionamos na introdução, na análise de Levin \& Rappaport-Hovav (1995), a distinção léxico-semântica relevante para a alternância causativa é a diferença que há entre verbos que denotam eventualidades causadas internamente e verbos que denotam eventualidades causadas externamente. Os verbos que participam da alternância causativa são os causados externamente - isto é, os verbos que envolvem, inerentemente, uma causa externa com controle sobre o evento causado. Já os verbos causados internamente teriam uma estrutura básica monoargumental, e por isso não participariam da alternância: de acordo com Levin \& Rappaport Hovav, o processo produtivo seria a formação da variante intransitiva do verbo a partir da variante transitiva. Levin \& Rappaport Hovav (1995) classificam, de um modo geral, os verbos que participam da alternância causativa em inglês como "verbos de mudança":

(12) a. Mudança de posição: bounce, move, roll, rotate, spin, ...

b. Mudança de estado: bake, blacken, break, close, cook, cool, dry, freeze, melt, open, shatter, thaw, thicken, whiten, widen, ...

(LEVIN \& RAPPAPORT HOVAV, 1995, p.93)

Segundo a análise das autoras, tanto os verbos de mudança de estado em (12b) como os verbos de modo de movimento não agentivos em (12a) são "verbos de mudança". Esta é, no entanto, uma hipótese complicada no que diz respeito à forma de representação léxico-semântica dos verbos de causação externa: a representação léxicosemântica proposta pelas autoras para os verbos que participam da alternância causativa, em (11), não reflete a semântica de maneira dos verbos em (12a): (11) representa tais 
verbos como "verbos de resultado" e os distingue dos verbos de maneira. Mas, como dissemos antes e como veremos na seção seguinte, é preciso que a representação semântica destes verbos os aproxime dos verbos de maneira.

Para este fim, nossa proposta é a de que outro predicado primitivo precisa ser adicionado à análise da estrutura léxico-semântica dos verbos: o predicado MOVE. MOVE foi introduzido por Jackendoff (1990) em sua teoria semântica para tratar de eventos que descrevem o movimento de um objeto, mas não implicam necessariamente deslocamento ou travessia de um trajeto. Esta é uma característica crucial dos verbos de modo de movimento, tanto agentivos como não agentivos - ou, nos termos de Jackendoff, volicionais e não volicionais:

(13) a. Debbie danced.

'Debbie dançou'

b. The flag waved.

'A bandeira tremulou'

(JACKENDOFF, 1990, p. 88)

Como não há uma trajetória inerente nestes eventos, a simples utilização da função GO, conforme a análise realizada em Jackendoff (1983), não seria possível, o que leva o autor a propor a seguinte função monoargumental para eventos deste tipo:

$$
\text { [Event } \operatorname{MOVE}([\text { Thing } x])]
$$

Nossa concepção de MOVE, contudo, é um tanto diferente da proposta por Jackendoff (1990). A distinção entre eventos - ou melhor, "ações" ou "movimentos" volicionais e não-volicionais no modelo proposto pelo autor é feita por meio de um traço modificador da função primitiva AFFECT, que define as relações de afetação no tier de ação. Em nossa análise, a volição é um elemento conceitual inerente ao predicado ACT. Mais precisamente, o predicado ACT é o predicado primitivo que está por trás de ações/movimentos volicionais - isto é, de qualquer evento concebido como um "agir"). Já MOVE, em nossa proposta, é o predicado primitivo que está por trás de ações/movimentos não volicionais - isto é, de qualquer evento concebido como um "mover-se". ${ }^{3}$

A nosso ver, a distinção entre verbos (de modo de movimento) com ACT e verbos com MOVE deve também ser a responsável pela oposição entre ações causadas internamente - que são "modos de agir" - e ações não (necessariamente) causadas internamente - que são "modos de mover-se (não volicionais)". Finalmente, ambos os predicados ACT e MOVE devem ser concebidos como "modos de movimento"

\footnotetext{
${ }^{3}$ Note-se que todo "agir" é um "mover-se", mas não vice-versa. Isso sugere que é possível analisar ACT como "MOVE volicional", o que aproximaria mais os verbos em (13a) e (13b). Esta é uma possibilidade que investigaremos no futuro.
} 
(volicional vs. não volicional) sem implicar necessariamente travessia de um trajeto sendo nisso distintos, por exemplo, do predicado GO, como para Jackendoff (1990).

Assim, para nós, sentenças como (15a) possuem uma representação léxicosemântica como (15b), e sentenças como (16a), uma representação como (16b):

(15) a. João caminhou/nadou/correu.

b. $\left[x \mathrm{ACT}_{<M A N N E R: \text { CAMINHAR, NADAR, etc. }>]}\right.$

(16) a. A bola rolou/quicou/girou.

b. $\left[x \mathrm{MOVE}_{<M A N N E R: \text { ROLAR, QUICAR, etc. }>]}\right]$

Eventos com causação interna são ou eventos que dependem de volição - por exemplo, eventos que envolvem ACT (cf. (15)) - ou eventos que envolvem processos inerentes ao participante (por exemplo, processos físicos não voluntários próprios dos seres animados, como florescer e enrubescer). Eventos com MOVE, como os em (16), são não volicionais e não envolvem um processo inerente ao participante; são, portanto, eventos sem causação interna. Por esta razão, presumimos que são mais facilmente concebíveis como tendo causação externa - diferentemente dos eventos envolvendo ACT (já que a causa externa deve afetar a volição do argumento de ACT para que o evento se realize). A nosso ver, esta é a explicação para o fato de que verbos de maneira com MOVE são capazes de participar de um processo lexical similar ao da alternância causativa; e, também, para o fato de que verbos de maneira com ACT, diferentemente, não participam produtivamente deste processo:

(17) a. João rolou/quicou/girou a bola.

b. $\quad\left[x \operatorname{CAUSE}\left[y \mathrm{MOVE}_{<M A N E R>}\right]\right]$

(18) a. João *caminhou/*nadou/\#correu Maria.

b. $*\left[x \operatorname{CAUSE}\left[x \mathrm{ACT}_{<M A N N E R>}\right]\right]$

Crucialmente, a representação léxico-semântica proposta para os verbos de modo de movimento em (16b) especifica que a raiz destes verbos expressa um modificador de maneira aplicado a um predicado de movimento primitivo - como os verbos em (15b). E, ao mesmo tempo, por MOVE ser um predicado primitivo que não envolve causação interna inerente, (16b) permite que verbos como rolar e girar, por exemplo, sejam distinguíveis dos verbos de ACT com respeito a processos de causação; assim, verbos com MOVE e com ACT podem ser tratados de modo diferente por processos lexicais como a causativização em português (que converte um predicado com a estrutura básica em (16b) em um evento causativo, como em (17b)).

$\mathrm{Na}$ seção seguinte, discutiremos a semântica do modificador quase. Nosso objetivo será o de mostrar que - em consonância com a teoria que acabamos de esboçar 
- verbos com MOVE e com ACT formam, de fato, uma classe natural quanto à estrutura de eventos, em contraste com os verbos de mudança de estado.

\section{O MODIFICADOR QUASE}

A ideia de que modificadores como quase e de novo são sensíveis a diferentes partes do significado verbal é bastante antiga na literatura, e foi introduzida na Semântica Gerativa, no final da década de 1960 e início da década de 1970, para justificar as estruturas semânticas propostas nesta teoria (cf. McCawley, 1971, 1973, e Morgan, 1969, apud Dowty, 1979). De acordo com Dowty (1979), a ambiguidade gerada pelo advérbio quase pode ser utilizada como teste para identificar a telicidade de um predicado. Sentenças como João quase desenhou um círculo seriam ambíguas entre uma leitura interna, na qual João começou a desenhar um círculo, mas não chegou a desenhá-lo por completo, e uma leitura externa, "contrafactual", na qual João esteve próximo de começar a desenhar, mas por alguma razão não deu início à ação. Por outro lado, sentenças atélicas como João quase correu não apresentam este tipo de ambiguidade: a única leitura possível é a "contrafactual".

Contrariamente a Dowty, Winter (2006) afirma que a ambiguidade gerada por quase não está relacionada necessariamente à telicidade dos predicados, mas sim à propriedade de denotarem escalas fechadas ou não. Com base no estudo de Rothstein \& Winter (2004) sobre pares de adjetivos totais e parciais, como limpo e sujo, e sua interação com advérbios como quase, Winter (2006) afirma que o modificador quase requer escalas fechadas. Consideremos os seguintes exemplos utilizados pelo autor:

(19) Dan almost walked around the lake.

'Dan quase andou ao redor do lago'

(20) Dan almost circled the lake.

'Dan quase contornou o lago'

(WINTER, 2006, p. 5)

Conforme Winter (2006), ambos os predicados modificados por almost em (19) e (20) são télicos, pois denotam eventos em que há uma trajetória com um ponto final definido. No entanto, em (20) observa-se a ambiguidade entre uma leitura na qual Dan iniciou a ação e chegou perto de contornar o lago - chamada pelo autor de "escalar" -, e uma leitura "contrafactual", na qual Dan esteve próximo de iniciar a atividade, mas não a iniciou. Em contraste, segundo o autor, a leitura escalar em (19) seria estranha para os falantes de inglês, e a interpretação mais natural seria a contrafactual, na qual a caminhada não é iniciada. Isso ocorreria porque a trajetória denotada pelo verbo em (20) seria fechada, ao contrário da trajetória em (19), introduzida pelo preposição around. 
A ideia de que o modificador quase é sensível a estruturas que denotam escalas fechadas vai ao encontro da proposta de Rappaport Hovav \& Levin (2010) em relação à distinção entre verbos de maneira e resultado. Salvo os casos de verbos de resultado que denotam escalas abertas - ou eventos com trajetórias télicas, porém abertas (cf. (19)) -, espera-se que verbos que denotam mudanças escalares apresentem ambiguidade com quase, enquanto verbos de maneira possibilitem somente a leitura contrafactual:

(21) a. Paulo quase cozinhou o arroz.

b. Paulo quase estancou o vazamento.

(22) a. Maria quase correu/nadou/caminhou.

b. João quase rolou/quicou/girou a bola.
Leitura escalar e leitura contrafactual

Leitura escalar e leitura contrafactual

Somente leitura contrafactual Somente leitura contrafactual

Crucialmente, os exemplos acima mostram que tanto os verbos de modo de movimento agentivos em (22a) como os não agentivos em (22b) apresentam somente a leitura contrafactual, na qual o evento não chegou a se realizar. Isto mostra que os verbos de movimento não agentivos comportam-se, com relação à estrutura de eventos detectada por quase, como os verbos de movimento agentivos: nos termos de Winter, ambos envolvem "escalas fechadas", diferentemente dos verbos de mudança de estado. Nos termos da análise que propusemos aqui, baseada nas propostas de RappaportHovav \& Levin, ambos são verbos de maneira, e diferem dos verbos de resultado contrariamente ao que propõem Rappaport-Hovav \& Levin para os verbos de modo de movimento não agentivos. Se considerarmos que a mudança escalar dos verbos de resultado advém da combinação do predicado BECOME e uma raiz de estado resultante, a modificação com quase serve como teste para mostrar que os verbos em (22) não possuem nem BECOME, nem um estado resultante inerente em seu significado - exatamente como em nossa análise.

Por fim, é importante observar que, ao introduzirmos uma trajetória fechada no evento, tanto os verbos de modo de movimento não agentivos como os agentivos apresentam a ambiguidade esperada, como mostram os exemplos em (23):

(23) a. João quase rolou a bola até a porta.

b. Paulo quase rodou o carro para fora da pista.

c. Maria quase correu/caminhou até a escola.

Todas as sentenças em (23) são ambíguas entre uma leitura escalar e uma leitura contrafactual, o que indica que a semântica de quase é sensível tanto à mudança de estado como ao movimento em uma trajetória: não importa a natureza da mudança, desde que ela seja escalar em uma escala fechada. 


\section{CONSIDERAÇÕES FINAIS}

Uma das principais consequências da proposta apresentada neste trabalho diz respeito aos tipos de predicados primitivos que as raízes dos verbos podem modificar. Conforme vimos, Rappaport Hovav \& Levin (2010) afirmam que as raízes podem tanto modificar o predicado ACT - no caso das raízes de maneira - como ser argumentos de BECOME - no caso das raízes de resultado. De acordo com nossa proposta, além destes casos previstos pelas autoras, as raízes de maneira também podem modificar o predicado MOVE, no caso dos verbos de modo de movimento não agentivos.

Mostramos também que o teste com o modificador quase indica que a representação léxico-semântica dos verbos de modo de movimento não agentivos assemelha-se à dos verbos de modo de movimento agentivo: a falta de ambiguidade no uso do quase sugere que ambas as classes possuem estrutura de eventos semelhantes; em nossa proposta, consistem em verbos de maneira - ou verbos que denotam eventos de mudança não escalar. Lembramos, ainda, que nossa proposta fornece uma chave para a participação dos verbos de modo de movimento não agentivos em um processo similar ao da alternância causativa: embora os verbos desta classe não sejam verbos de mudança de estado (contrariamente ao que propõem Levin \& Rappaport Hovav (1995)), sua raiz de maneira modifica o predicado primitivo MOVE: este é um predicado não volicional e que não envolve causação interna, o que possibilita mais facilmente a conceitualização destes eventos como causados externamente.

\section{REFERÊNCIAS}

DOWTY, D. Word Meaning and Montague Grammar: the semantics of verbs and times in Generative Semantics and in Montague's PTQ. Dordrecht: Reidel, 1979.

FILIP, H. \& ROTHSTEIN, S. Telicity as a semantic parameter. In: Lavine, J., Franks, S., Tasseva-Kurktchieva, M. \& Filip, H. (eds.). Formal Approaches to Slavic Linguistics: The Princeton Meeting 2005. Ann Arbor, MI: Michigan Slavic Publications, University of Michigan, 2006, 139-56.

JACKENDOFF, R. Semantics and Cognition. Cambridge, MA: MIT Press, 1983.

Semantic Structures. Cambridge, MA: MIT Press, 1990.

KRIFKA, M. The origins of telicity. In: Rothstein, S. (ed.). Events and Grammar. Dordrecht: Kluwer Academic Publishers, 1998, 197-236.

LEVIN, B. \& RAPPAPORT HOVAV, M. Unaccusativity: At the Syntax-Lexical Semantics Interface. Cambridge, MA: MIT Press, 1995.

. Argument Realization. Cambridge: CUP, 2005.

RAPPAPORT HOVAV, M. \& LEVIN, B. Building Verb Meanings. In: Butt, M. \& Geuder, W. (eds.). The Projection of Arguments: Lexical and Compositional Factors. Stanford: CSLI Publications, 1998, p. 97-134. 
Reflections on Manner/Result Complementarity. In: Doron, E., Rappaport Hovav, M. \& Sichel, I. (eds.). Syntax, Lexical Semantics, and Event Structure. Oxford: Oxford University Press, 2010, p. 21-38.

ROTSTEIN, C. \& WINTER, Y. Total adjectives vs. partial adjectives: scale structure and higher-order modification. Natural Language Semantics, 12:259-288, 2004.

WINTER, Y. Closure and telicity across categories. In: Tancredi, C., Kanazawa, M., Imani, I., \& Kusumoto, K. (eds.). Proceedings of Semantics and Linguistic Theory. SALT16, 2006. 